\title{
ORIGINAL
}

\section{LA EDAD COMO ESCALA DE TIEMPO EN EL ANÁLISIS DE LA SUPERVIVENCIA POR TRASTORNOS CRÓNICOS (*)}

\section{Cristina Rius Gibert y Gloria Pérez Albarracín por el grupo Cohesca.}

Agencia de Salud Pública de Barcelona, Barcelona.

(*) Este estudio fue financiado parcialmente por el Fondo de Investigación Sanitaria (FIS 98/0053-01) y forma parte de los trabajos para la obtención del doctorado en Salud Pública e Investigación Biomédica de la Universidad Autónoma de Barcelona de Cristina Rius.

\section{RESUMEN}

Fundamento: El análisis de supervivencia de estudios de cohortes no tiene en cuenta la edad a la que un individuo muere sino durante el cual ha estado siendo incluido en el seguimiento. El objetivo de este trabajo es valorar las diferencias que resultan en el cálculo del riesgo relativo cuando se utiliza la edad en lugar de seguimiento como escala de tiempo en el análisis de supervivencia de los trastornos crónicos.

Métodos: Se analizan los datos de la cohorte de seguimiento de 5 años de la encuesta de salud de Cataluña (COHESCA). Se ajustan modelos de Cox con el tiempo de seguimiento (enfoque «habitual») y la edad (enfoque «alternativo») como escala de tiempo. Como principal covariable se usa el índice de comorbilidad y se ajusta por variables demográficas, estilos de vida y salud auto-percibida.

Resultados: En los hombres de 40-64 años en el enfoque "habitual" no se observa aumento significativo del riesgo relativo de morir. En las mujeres de 40-64 años con puntuación 3-4 del índice hay un incremento significativo del riesgo relativo. En las personas mayores de 64 años se observa un aumento significativo del riesgo para la puntuación 3-4 [RRhombres=2,1 (1,3-2,5); RRmujeres $=2,1(1,2-3,7)]$ y $>=5$ del índice (RRhombres=3,4 (1,7-6,9); RRmujeres=4,0 (2,1-7,7). En el enfoque «alternativo», el riesgo relativo fue de $1,0(0,7-1,4)$ en los hombres con una puntuación de 1-2, 1,5 (1,0-2,3) para los de 3-4 y $2,6(1,4-4,9)$ para los de $>=5$. En las mujeres, los RR fueron respectivamente $0,8(0,5-1,2), 1,7(1,1-2,7)$ y $2,6(1,5-4,8)$

Conclusiones: Ambos enfoques muestran un aumento del riesgo relativo según el índice de comorbilidad. Sin embargo, en el enfoque «alternativo», se ajusta por el efecto confundidor de la edad.

Palabras clave: Estudios de cohortes. Modelos de riesgos proporcionales. Análisis de supervivencia. Trastornos crónicos. Factores confusores.

Correspondencia:

Cristina Rius MD, MPH

Agencia de Salud Pública de Barcelona

Plaça Lesseps, 1

08023 Barcelona, Spain.

Correo electrónico: 32284mrg@comb.es
ABSTRACT

\section{Age as Time-Scale: An Application to the Survival Analysis of Chronic Diseases}

Background: Cohort study survival analysis does not take into account the age at which an individual dies, but rather the age throughout which the individual has been included in the follow-up. This study is aimed at assessing the resulting differences in the calculation of the relative risk (RR) when the age is used instead of follow-up as a timescale in the chronic diseases survival analysis.

Methods: The five-year cohort follow-up data for the Catalan Health Interview Survey (COHESCA) is analyzed. Cox models are adjusted to the follow-up time («standard» approach) and the age («alternative» approach) as the timescale. The main covariable used is the comorbility index, which is adjusted by demographics, lifestyles and self-perceived health.

Results: Among men in the 40-64 age range, using the «standard» approach, no significant increase in the RR of death was found. Among women within the 40-64 age range with a 3-4 score on the index, there was a significant increase in the RR. Among those over age 64 , a significant increase in the risk was found for the 3-4 score $(R R m e n=2.1(1.3-2.5)$; RRwomen $=2.1(1.2-3.7))$ and $>=5$ on the index $(\mathrm{RRmen}=3.4(1.7-6.9)$; RRwomen=4.0(2.1-7.7)). Using the «alternative» approach, the RR was 1.0 (0.7-1.4) among men with a score of 1-2, $1.5(1.0-2.3)$ for those with a score of 3-4 and 2.6 (1.4-4.9) for those with a score of $>=5$. Among women, the RR were respectively $0.8(0.5-1.2), 1.7(1.1-2.7)$ and $2.6(1.5-4.8)$.

Conclusions: Both approaches showed an increase in the RR according to the comorbility index. However, in the «alternative» approach, it is adjusted by the confounding effect of age.

Key words: Cohort studies. Proportional hazard models. Survival analysis. Chronic diseases cofounder. 


\section{INTRODUCCIÓN}

Actualmente, en las sociedades llamadas desarrolladas se está observando un incremento de la morbilidad entendida como la presencia de múltiples trastornos crónicos en una misma persona, siendo éste un problema de salud pública cada vez más frecuente $^{1}$. Sin embargo, la importancia del estudio de estos trastornos no sólo radica en la elevada prevalencia sino también en la gravedad de los trastornos crónicos que padece un determinado individuo ${ }^{2}$. Los índices de comorbilidad combinan el número de trastornos crónicos presentes en un solo individuo junto con su gravedad. Al mismo tiempo los índices de comorbilidad son buenos predictores del riesgo de morir, ya que además de tener en cuenta el número de trastornos crónicos que padece un individuo, también incluyen la gravedad de cada trastorno crónico ${ }^{3-5}$ en el riesgo de morir.

Los trastornos crónicos se comportan de manera diferente según la edad de los individuos en el sentido que en las personas más mayores coexisten más de un problema de salud, mientras que en individuos de mediana edad se presenta con frecuencia sólo uno ${ }^{6}$. En los trabajos que estudian el efecto de la presencia de múltiples trastornos crónicos sobre el riesgo de morir es necesario tomar en consideración la edad ya que está relacionada tanto con el aumento del número de trastornos crónicos como con el riesgo de morir. En los análisis de supervivencia si se tiene en cuenta un enfoque «habitual»se considera el tiempo de supervivencia como el periodo comprendido entre la inclusión de un determinado individuo en el estudio y la aparición del acontecimiento de interés, en este caso la muerte. Al ajustar por la edad se controla su efecto sobre el riesgo de morir sin tener en cuenta la edad a la que un individuo muere.

En un enfoque «alternativo» del análisis de supervivencia se considera la edad como escala de tiempo de supervivencia en lugar de utilizar el tiempo de seguimiento ${ }^{7}$. Con este enfoque se tienen en cuenta los factores que pueden modificar el riesgo de morir a partir de una determinada edad. Así por ejemplo, el hecho de que un individuo entre en un estudio a la edad de 40 años tendrá un efecto diferente sobre el riesgo de morir que si se incorpora a los 75 años, además de ajustar por el posible efecto confusor de la edad.

El objetivo de este trabajo es analizar la supervivencia en función de los trastornos crónicos en una cohorte representativa de la población de Cataluña utilizando dos escalas de tiempo distintas: el tiempo de seguimiento en el estudio y la edad de los participantes.

\section{SUJETOS Y MÉTODOS}

\section{Muestra y diseño del estudio}

La Encuesta de Salud de Cataluña (ESCA) del año 1994 fue un estudio transversal realizado por el Servicio Catalán de la Salud en una muestra representativa de la población no institucionalizada de Cataluña ${ }^{8}$. La encuesta incluyó información socio-demográfica así como sobre trastornos crónicos, estilos de vida, estado de salud autopercibido, uso de servicios sanitarios, prácticas preventivas, discapacidades y salud mental.

Se seleccionó a 15.000 individuos a partir de un muestreo aleatorio polietápico. El cuestionario se administró por encuestadores entrenados entre enero y diciembre de 1994. Sólo un 5,4\% de los individuos seleccionados fueron sustituidos por ausencia o por rechazo a contestar. Para este estudio sólo fueron incluidos los 6.641 individuos de la muestra inicial que tenían entre 40 y 84 años en el momento de realizar la encuesta (1994).

Para establecer el seguimiento se obtuvo el estado vital a través de una conexión de registros con el Registro de Mortalidad de 
Cataluña (RMC) para cada año desde 1994 hasta 1998. Así pues, el inicio del seguimiento corresponde a la fecha de la encuesta y el final del seguimiento a la fecha de la muerte o la fecha de censura (31 de diciembre de 1998). El proceso de conexión así como el trabajo realizado fue aprobado por la Comisión de manejo de información confidencial del Departamento de Sanidad y Seguridad Social y se respetó la privacidad y la confidencialidad.

\section{Variables de estudio}

La variable dependiente fue el estado vital. La variable independiente principal fue el índice de comorbilidad, cuya forma de obtención se explica ampliamente en otra publicación $^{5}$. En resumen, este índice se obtiene adaptando la metodología de Charlson et al. ${ }^{3}$ como el sumatorio de los trastornos crónicos declarados multiplicados por el peso correspondiente al riesgo relativo de morir de cada uno de los trastornos crónicos. La encuesta incluyó información sobre la presencia de 16 trastornos crónicos: hipertensión, enfermedades del corazón, varices, artrosis, alergias, asma, bronquitis, diabetes, úlcera péptica, molestias urinarias, colesterol elevado, cataratas, alteraciones de la piel, estreñimiento, depresión y embolia

El resto de variables explicativas fueron el sexo, la clase social agrupada en 3 categorías basadas en la Clasificación Nacional de Ocupaciones $^{9}$ : clase I-II, clase III y clase IV$\mathrm{V}$, y las variables de los estilos de vida, los cuales se agruparon de la siguiente forma: el consumo de tabaco en 3 categorías según los criterios de la OMS (fumador actual, ex fumador y no fumadores); el consumo de alcohol en 4 categorías: abstemios y según los terciles de consumo por sexo $(9,60 \mathrm{~g} / \mathrm{día}$ y 20,80 g/día en hombres y 3,20 g/día y 10,97 g/día para las mujeres). La actividad física fue obtenida a partir de la información de la actividad física habitual y la del tiempo libre y quedó agrupada en 2 categorías (acti- vos y no activos). El estado de salud autopercibido fue obtenido directamente y agrupado en 3 categorías: excelente y muy buena, buena, y regular y mala.

\section{Análisis estadístico}

Para obtener la relación entre las covariables y la mortalidad se ajustó un modelo de riesgos proporcionales de Cox con el tiempo de seguimiento en el análisis «habitual»y con la edad como escala de tiempo en el enfoque «alternativo». Como principal covariable se utilizó el índice de comorbilidad y se ajustó por las variables explicativas.

Con el fin de eliminar el efecto confusor de la edad, en el análisis de supervivencia «alternativo» se consideró ésta como escala de tiempo en lugar del tiempo de seguimiento. Así, aquellos individuos que entraron en el estudio a una edad superior a los 40 años entraron «tarde» en el estudio y fueron considerados como datos truncados por la izquierda. Además, aquellos individuos que estaban vivos al final del estudio fueron considerados datos censurados por la derecha ${ }^{10}$. En este estudio los datos eran truncados por la izquierda y censurados por la derecha y fue, por tanto, necesario utilizar una extensión del modelo de riesgos proporcionales que incorpora las entradas retrasadas (figura 1). Así, si un individuo se incluye en el estudio a los 45 años el tiempo de seguimiento será hasta los 49 años si no se muere durante el periodo de seguimiento. De esta forma obtendríamos la probabilidad de morir a una edad determinada, por ejemplo a los 50 años de edad en lugar de a los cinco años de seguimiento. En este enfoque, el número de individuos en riesgo podía fluctuar de manera dinámica con el tiempo en lugar de seguir una tendencia decreciente ${ }^{11,12}$.

Las variables con más de 2 categorías se incluyeron como «dummy» usando como categoría de referencia la de menor riesgo. 
Figura 1

Edad como escala de tiempo. ( |: Edad a la entrada en el estudio, x: Edad a la salida del estudio, o: Edad debida a la censura por la derecha).

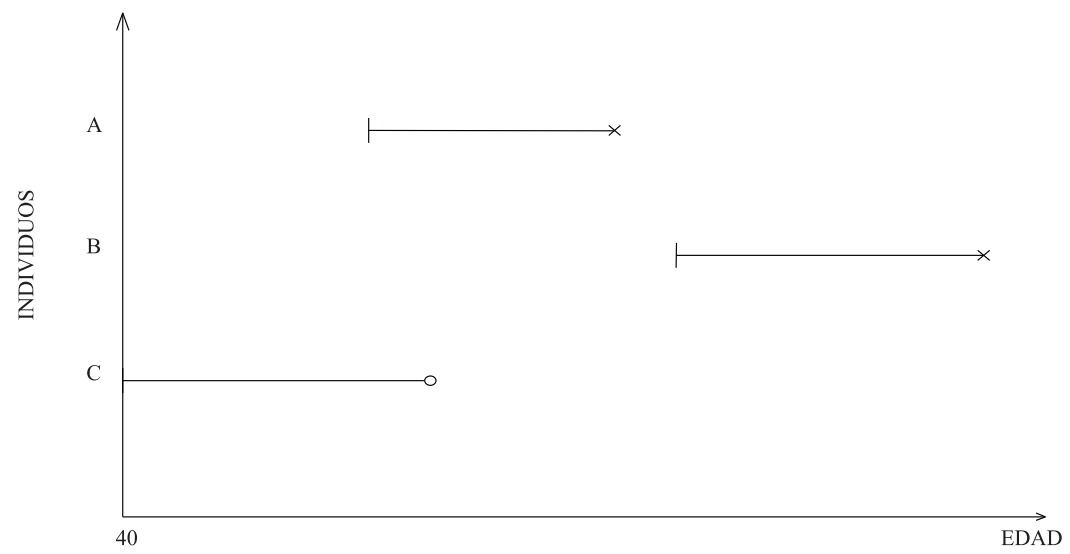

Se comprobó la proporcionalidad de las variables tanto gráfica como estadísticamente. Se exploraron distintos términos de interacción con el fin de detectar posibles modificaciones del efecto, pero ninguno de ellos resultó estadísticamente significativo y por tanto no fueron incluidos en los modelos.

Los modelos se ajustaron paso a paso para controlar posibles efectos de multicolinaeridad. Como prueba estadística general se utilizó la de la máxima verosimilitud observada cuando cada variable era incluida en el modelo.

Todo el análisis se realizó estratificando por sexos y aplicando los pesos derivados del proceso de estratificación muestral ${ }^{13}$. El análisis se realizó con los paquetes estadísticos SPSS versión 11.0 y Stata (versión 9.0, Texas: College Station; 2005).

\section{RESULTADOS}

De los 6.641 individuos que tenían entre 40 y 84 años al comenzar el estudio, 3.105 eran hombres y 3.536 mujeres, y se contabilizaron 386 muertes al final del seguimiento (234 hombres y 152 mujeres).
La mediana de edad al inicio fue de 70 años para los hombres fallecidos y de 56 entre los hombres considerados censuras. En las mujeres muertas la mediana de edad al inicio fue de 71 años, y de 57 en las mujeres censuradas. La mediana de tiempo de seguimiento de los casos que murieron fue de 2,83 años en los hombres y de 2,41 en las mujeres; para los casos censurados la mediana de tiempo de seguimiento fue de 4,51 años tanto en hombres como en mujeres. En la tabla 1 se muestra la relación de las variables de nivel socioeconómico, de estilos de vida y de salud percibida con el estado vital a los 5 años de seguimiento y estratificado por sexo. Cabe destacar que todas las variables mantienen una asociación significativa con el estado vital excepto la clase social en los hombres. A pesar de ello, todas las variables se utilizaron en el modelo ajustado.

En la tabla 2 se muestran los RR de morir a los 5 años según las categorías del índice de comorbilidad para el enfoque «habitual»y para el enfoque «alternativo». En el enfoque «habitual», incluyendo la edad como una de las variables de ajuste, el RR de morir en los hombres con una puntuación del índice de comorbilidad 1-2 respecto a los que tenían un índice de 0 fue de 1,02 (0,74-1,41); el RR 
Tabla 1

Descripción de la cohorte de 40 a 84 años según estado vital en hombres $(\mathrm{N}=3.105)$ y en mujeres $(\mathrm{N}=3.536)$. Estudio Cohesca, 1994-1998

\begin{tabular}{|c|c|c|c|c|c|c|}
\hline & \multicolumn{3}{|c|}{ HOMBRES } & \multicolumn{3}{|c|}{ MUJERES } \\
\hline & Vivos $(\mathrm{n}=2.871)$ & Muertos ( $\mathrm{n}=234)$ & Valor $\mathrm{p}$ & Vivas $(\mathrm{n}=3.384)$ & $\begin{array}{l}\text { Muertas } \\
(\mathrm{n}=152)\end{array}$ & Valor $\mathrm{p}$ \\
\hline & $\%(n)$ & $\%(n)$ & & $\%(n)$ & $\%(n)$ & \\
\hline \multicolumn{7}{|l|}{ Edad agrupada (años) } \\
\hline $40-64$ & $97,0(2137)$ & $3,0(67)$ & $<0,01$ & $98,0(2359)$ & $2,0(49)$ & 0,01 \\
\hline $65-84$ & $81,5(734)$ & $18,5(167)$ & & $91,0(1026)$ & $9,0(102)$ & \\
\hline \multicolumn{7}{|l|}{ Clase social } \\
\hline I-II & $94,4(665)$ & $5,6(39)$ & 0,069 & $96,1(572)$ & $3,9(23)$ & 0,01 \\
\hline III & $92,2(558)$ & $7,8(47)$ & & $98,7(418)$ & $1,3(5)$ & \\
\hline IV-V & $91,8(1648)$ & $8,2(148)$ & & $95,1(2395)$ & $4,9(123)$ & \\
\hline \multicolumn{7}{|l|}{ Consumo de tabaco $\dagger$} \\
\hline No Fumador & $94,6(801)$ & $5,3(44)$ & $<0,01$ & $95,4(2941)$ & $4,6(141)$ & 0,039 \\
\hline Ex-fumador & $89,9(987)$ & $10,1(109)$ & & $95,9(161)$ & $4,1(7)$ & \\
\hline Fumador & $93,3(1082)$ & $6,7(77)$ & & $98,6(283)$ & $1,4(4)$ & \\
\hline \multicolumn{7}{|l|}{ Consumo de alcohol $\dagger+$} \\
\hline Abstemio & $89,0(939)$ & $11,0(117)$ & $<0,01$ & $94,8(2261)$ & $5,2(124)$ & 0,01 \\
\hline $1 \mathrm{r}$ tercil & $93,6(655)$ & $6,4(45)$ & & $96,6(437)$ & $3,4(16)$ & \\
\hline $2^{\circ}$ tercil & $93,4(632)$ & $6,6(45)$ & & $98,6(302)$ & $1,4(4)$ & \\
\hline $3 \mathrm{r}$ tercil & $95,8(644)$ & $4,2(28)$ & & $98,0(384)$ & $2,0(8)$ & \\
\hline \multicolumn{7}{|l|}{ Actividad física $\dagger$} \\
\hline Activos & $93,9(1865)$ & $6,1(122)$ & $<0,01$ & $96,7(1866)$ & $3,3(64)$ & 0,01 \\
\hline No activos & $89,9(1005)$ & $10,1(112)$ & & $94,5(1518)$ & $5,5(88)$ & \\
\hline \multicolumn{7}{|l|}{ Estado de salud auto percibido } \\
\hline Excelente+muy buena & $97,2(395)$ & $2,8(12)$ & $<0,01$ & $98,5(325)$ & $1,5(5)$ & 0,01 \\
\hline Buena & $94,6(1636)$ & $5,4(93)$ & & $97,4(1529)$ & $2,6(41)$ & \\
\hline Regular+mala & $86,6(839)$ & $13,4(130)$ & & $93,5(1530)$ & $6,5(106)$ & \\
\hline \multicolumn{7}{|l|}{ Índice de Comorbilidad } \\
\hline 0 & $95,4(1649)$ & $4,6(79)$ & $<0,01$ & $97,0(2022)$ & $3,0(62)$ & 0,01 \\
\hline $1-2$ & $91,2(999)$ & $8,8(96)$ & & $96,0(1026)$ & $4,0(43)$ & \\
\hline $3-4$ & $81,6(200)$ & $18,4(45)$ & & $89,4(261)$ & $10,6(31)$ & \\
\hline$>=5$ & $62,2(23)$ & $37,8(14)$ & & $82,4(75)$ & $17,6(16)$ & \\
\hline
\end{tabular}

Aplicada la ponderación derivada del muestreo. $†$ No se pregunta a los incapacitados para responder $\ddagger$ Límites de los intervalos en g/día. Hombres: cat1:<9.6, cat2: 9.6-20.8, cat3: >20.8 . Mujeres: cat1:<3.2, cat2: 3.2-10.97, cat3:>10.97. 
Tabla 2

Mortalidad según las categorías del índice de comorbilidad (Riesgos relativos (RR) e Intervalos de Confianza del $95 \%$ (IC 95\%)) utilizando el método habitual con la edad como covariable y el método alternativo con la edad como escala de tiempo. Estudio Cohesca, 1994-1998

\begin{tabular}{|c|c|c|c|c|c|c|}
\hline \multirow[b]{2}{*}{ Método habitual } & \multicolumn{3}{|c|}{ Hombres $(n=3.105)$} & \multicolumn{3}{|c|}{ Mujeres (n=3.536) } \\
\hline & $\begin{array}{l}\text { Índice de } \\
\text { Comorbilidad }\end{array}$ & $\mathrm{RR} \dagger$ & IC $95 \%$ & $\begin{array}{l}\text { Índice de } \\
\text { Comorbilidad }\end{array}$ & $\mathrm{RR} \dagger$ & IC $95 \%$ \\
\hline & 0 & 1,0 & & 0 & 1,0 & \\
\hline & $1-2$ & 1,02 & $0,74-1,41$ & $1-2$ & 0,85 & $0,57-1,27$ \\
\hline & $3-4$ & 1,59 & $1,0-2,40$ & $3-4$ & 1,82 & $1,16-2,87$ \\
\hline & $>=5$ & 2,94 & $1,60-5,41$ & $>=5$ & 2,84 & $1,60-5,04$ \\
\hline \multirow[t]{5}{*}{$40-64$ años } & $\begin{array}{l}\text { Índice de } \\
\text { Comorbilidad }\end{array}$ & RRt† & IC $95 \%$ & $\begin{array}{l}\text { Índice de } \\
\text { Comorbilidad }\end{array}$ & RR†T & IC $95 \%$ \\
\hline & 0 & 1,0 & & 0 & 1,0 & \\
\hline & $1-2$ & 0,80 & $0,45-1,43$ & $1-2$ & 0,86 & $0,43-1,71$ \\
\hline & $3-4$ & 1,69 & $0,78-3,67$ & $3-4$ & 2,23 & $1,04-4,78$ \\
\hline & $>=5$ & 3,02 & $0,85-10,67$ & $>=5$ & 1,25 & $0,24-6,43$ \\
\hline \multirow[t]{5}{*}{65 años y más } & $\begin{array}{l}\text { Índice de } \\
\text { Comorbilidad }\end{array}$ & $R R+\dagger$ & IC $95 \%$ & $\begin{array}{l}\text { Índice de } \\
\text { Comorbilidad }\end{array}$ & $\mathrm{RR}+\dagger$ & IC $95 \%$ \\
\hline & 0 & 1,0 & & 0 & 1,0 & \\
\hline & $1-2$ & 1,33 & $0,90-1,98$ & $1-2$ & 0,93 & $0,57-1,52$ \\
\hline & $3-4$ & 2,11 & $1,28-3,45$ & $3-4$ & 2,11 & $1,20-3,73$ \\
\hline & $>=5$ & 3,37 & $1,66-6,86$ & $>=5$ & 4,03 & $2,11-7,71$ \\
\hline \multirow[t]{5}{*}{ Método alternativo } & $\begin{array}{l}\text { Índice de } \\
\text { Comorbilidad }\end{array}$ & $\mathrm{RR}+\dagger$ & IC $95 \%$ & $\begin{array}{l}\text { Índice de } \\
\text { Comorbilidad }\end{array}$ & $\mathrm{RR} \dagger$ & IC $95 \%$ \\
\hline & 0 & 1,0 & & 0 & 1,0 & \\
\hline & $1-2$ & 1,02 & $0,74-1,41$ & $1-2$ & 0,83 & $0,56-1,24$ \\
\hline & $3-4$ & 1,51 & $1,0-2,30$ & $3-4$ & 1,72 & $1,08-2,72$ \\
\hline & $>=5$ & 2,65 & $1,43-4,89$ & $>=5$ & 2,65 & $1,48-4,77$ \\
\hline
\end{tabular}

$\dagger \quad$ RR Ajustado por edad, clase social, habito tabáquico, consumo de alcohol, actividad física y estado de salud autopercibido.

$\dagger \dagger$ RR Ajustado por clase social, habito tabáquico, consumo de alcohol, actividad física y estado de salud autopercibido.

aumentó hasta $1,59(1,05-2,40)$ en la categoría 3-4 y hasta 2,94 (1,60-5,41) en la categoría de puntuación 5 o mayor. En las mujeres los RR de morir en la primera categoría fue de $0,85(0,57-1,27)$, incrementándose hasta 1,82 $(1,16-2,87)$ en la segunda categoría y hasta $2,84(1,60-5,04)$ en la última categoría.
En el enfoque habitual y estratificando por la edad el RR de morir no presenta ningún incremento significativo en relación al índice de comorbilidad en los hombres de 40 a 64 años. En las mujeres de 40 a 64 años sólo muestra una relación siginificativa la puntuación 3-4 del índice. 
En los mayores de 64 años, tanto en hombres como en mujeres, se observó un aumento significativo del riesgo para la puntuación 3-4 $\left(\mathrm{RR}_{\text {hombres }}=2,11 ; 1,28-2,45 ; \mathrm{RR}_{\text {muje- }}\right.$ res $=2,11 ; 1,20-3,73)$ y 5 o más del índice $\left(\mathrm{RR}_{\text {hombres }}=3,37 ; 1,66-6,86 ; \mathrm{RR}_{\text {mujeres }}=4,03\right.$; 2,11-7,71).

En el enfoque «alternativo», una vez ajustado por el resto de covariables, el RR de morir fue de 1,02 (0,73-1,41) en los hombres con una puntuación del índice de comorbilidad de 1-2 respecto a los que tenían un índice de 0 ; en la categoría 3-4 de comorbilidad el RR fue de 1,51 (1-2,30) y aumentó hasta 2,64 (1,43-4,89) cuando la puntuación fue 5 o más. En las mujeres los RR de morir fueron respectivamente $0,83(0,55-1,24), 1,71$ $(1,09-2,72)$ y $2,65(1,47-4,77)$.

\section{DISCUSIÓN}

Para ambos sexos en los dos enfoques se observó un aumento del RR de morir para cada una de las categorías del índice de comorbilidad respecto a la de referencia (puntuación cero del índice de comorbilidad), sin embargo no se detectaron diferencias importantes en el valor de los RR entre las dos alternativas. En otro estudio realizado en una población de mayores de 65 años, los autores confirman que los riesgos de morir no varían sustancialmente cuando son usados estos dos enfoques ${ }^{7}$. Sin embargo, utilizar la edad como escala de tiempo en el análisis de supervivencia aporta ventajas respecto al enfoque «habitual». En primer lugar, se tiene en cuenta la edad a la que un determinado individuo entra en el estudio y el periodo durante el cual es seguido, es decir, cinco años de seguimiento pueden tener diferentes implicaciones dependiendo de la edad a la que el individuo haya sido incluido. Así por ejemplo, un individuo que entra en el estudio a los 45 años será seguido como mucho hasta los 50, mientras que uno de 75 años será seguido como máximo hasta los 80 y por tanto tendrán diferentes repercu- siones por lo que respecta al riesgo de morir. De esta forma, se estiman las probabilidades de morir en grupos de pacientes de la misma edad teniendo en cuenta una mayor homogeneidad de los factores de riesgo ${ }^{7}$.

Además, la edad simplifica el análisis en el sentido de reducir el número de modelos a ajustar y de facilitar la interpretación de los resultados. Cuando se utiliza el enfoque «habitual» el análisis se realiza bien incluyendo la edad como variable de ajuste bien realizando análisis separados para cada grupo de edad. En el enfoque «alternativo», utilizando la edad como escala de tiempo, se ajusta automáticamente por el efecto confusor de la edad, y además permite obtener la probabilidad de morir de individuos de una edad determinada en lugar de la probabilidad de los individuos que tienen un tiempo de seguimiento determinado.

En este trabajo hay que tomar en consideración varias limitaciones. En primer lugar, aquellas que son propias del diseño del estudio como es, por ejemplo, cierto grado de infradeclaración en determinadas variables recogidas en la encuesta ${ }^{14}$, o errores derivados de la conexión de los registros ${ }^{15}$. Respecto al análisis estadístico hay que tener en cuenta que no consideramos los cambios que se pueden producir en algunas variables a lo largo del tiempo ${ }^{16}$. Según Lamarca y cols ${ }^{7}$ en aquellos estudios donde no se puede actualizar la información de estas variables se recomienda reducir el seguimiento a la duración en la que se puede asumir que la exposición no variará sustancialmente. Así, en nuestro estudio el periodo de seguimiento es suficientemente corto (5 años) como para que podamos asumir la estabilidad de las variables de los estilos de vida. Por otro lado, hemos de asumir la posibilidad de que aquellos pacientes especialmente graves no hayan sobrevivido suficiente para que los hayamos podido incluir en el estudio y, por tanto, que haya cierta infraestimación del riesgo. Finalmente, utilizar la edad como escala de tiempo condiciona el hecho que los 
individuos en riesgo antes de la edad a la cual entraron en el estudio no sean tenidos en cuenta y por tanto el riesgo puede estar infraestimado.

En conclusión, el enfoque «alternativo» simplifica los resultados del análisis de supervivencia y proporciona una interpretación más apropiada en aquellas poblaciones donde interesa conocer el efecto de ciertos factores sobre el riesgo de morir a partir de una determinada edad, como por ejemplo el efecto de los trastornos crónicos sobre el riesgo de morir a partir de los 40 años.

\section{AGRADECIMIENTOS}

Agradecemos la colaboración de Rosa Lamarca.

\section{Grupo Cohesca}

Mara Bares, Departamento de Salud; Esteve Fernández, Unidad de Control y prevención del Cáncer. Instituto Catalán de Oncología; Montse García, Unidad de Control y prevención del Cáncer. Instituto Catalán de Oncología; Rosa Gispert, Departamento de Salud; Glòria Pérez, Agencia de Salud Pública de Barcelona; Cristina Rius, Agencia de Salud Pública de Barcelona; Anna Schiaffino, Unidad de Control y prevención del Cáncer. Instituto Catalán de Oncología; Ricard Tresserras, Departamento de Salud.

\section{BIBLIOGRAFÍA}

1. Guralnik JM. Assessing the impact of co-morbidity in older population. Ann Epidemiol 1996;6:376-80.

2. Ruigómez A, Alonso J, Antò JM. Relationship of health behaviours to five-year mortality in elderly cohort. Age Ageing 1995 ;24:113-9.

3. Charlson ME, Pompei P, Ales KL, Mackenzie CR. A new method of classifying prognostic comorbidity in longitudinal studies: development and validation. J Chron Dis 1987; 40(5): 373-83.
4. D'Hoore W, Bouckaert A, Tilquin C. Practical considerations on the use of the Charlson comorbidity index with administrative data bases. J Clin Epidemiol 1996; 49(12): 1429-33.

5. Rius C, Pérez G, Martínez JM, Barés M, Schiaffino A, Gispert R, Fernández E. The Charlson comorbidity index predicted subsequent mortality in a health survey. J Clin Epidemiol 2004; 57: 403-8.

6. Cornoni-Huntley JC, Foley DJ, Guralnik JM. Comorbidity analysis: a strategy for understanding mortality, disability and use of health care facilities of older people. Int J Epidem 1991; 20(1): S8-S17.

7. Lamarca R, Alonso J, Gómez G, Muñoz A. Lefttruncated data with age as time scale: an alternative for survival analysis in the elderly population. J Gerontol 1998; 53A(5): M337-M343.

8. Departament de Sanitat i Seguretat Social. Document tècnic. Enquesta de Salut de Catalunya (ESCA). Barcelona: Generalitat de Catalunya; 1996.

9. Grupo de trabajo de la SEE y de la SEMFyC. Una propuesta de medida de la clase social. Aten Primaria. 2000;25:350-63.

10. Klein JP, Moeschberger ML. Survival analysis: techniques for censored and truncated data. New York: Springer, cop; 1997.

11. Therneau TM, Grambsch PM. Modelling survival data. Extending the Cox model. New York: Springer-Verlag; 2000.

12. Andersen PK, Borgan O, Gill RD, Keiding N. Statistical models based on counting processes. New York: Springer-Verlag; 1993.

13. Guillen M, Junca S, Rue M, Aragay JM. Efecto del diseño de las muestras en el análisis de encuestas complejas: aplicación a la encuesta de salud de Cataluña. Gac Sanit 2000;14(5):399-402.

14. Lantz PM, House JS, Lepkowski JM, Williams DR, Mero RP, Chen J. Socioeconomic factors, health behaviours and mortality. JAMA 1998; 279: 170308 .

15. Mulder M, Ranchor AV, Sanderman R, Bouma J, Van der Heuvel WJA. The stability of lifestyle behaviour. Int J Epidemiol 1998; 27: 199-207.

16. Brenner H, Schmidtmann I, Stegmaier C. Effects of record linkage errors on registry-based follow-up studies. Statist Med 1997; 16: 2633-43. 